\title{
Escala de Motivação Docente: desenvolvimento e validação*
}

\section{Teacher Motivation Scale: development and validation}

\author{
Tárcia Rita Davoglio** \\ Bettina Steren dos Santos ${ }^{* *}$
}

\begin{abstract}
RESUMO
Este estudo teve por objetivo analisar evidências psicométricas de validade e confiabilidade da Escala de Motivação Docente (EMD), que visa explorar a motivação autodeterminada de professores. A EMD foi desenvolvida fundamentando-se nos pressupostos da Self-Determination Theory (SDT), que aborda a motivação autodeterminada como um processo intrínseco, sujeita a influências sociocontextuais a partir das necessidades psicológicas básicas de autonomia, competência e pertencimento. Após a análise de conteúdo e da semântica dos itens e o teste piloto, a versão preliminar da EMD foi aplicada em 398 professores universitários brasileiros. Os resultados exploratórios levaram ao refinamento da EMD, ficando a versão final composta por 24 itens, pontuados em uma escala do tipo Likert, distribuídos em cinco dimensões: Performance, Desenvolvimento, Prática Docente, Formação Continuada e Inserção Institucional. A análise fatorial confirmatória evidenciou índices de ajustes adequados, confirmando o modelo estrutural proposto. Ainda que incipientes, esses resultados são consistentes para evidenciar a validade de construto da EMD, apontando perspectivas para a sua utilização em avaliação motivacional docente. Recomenda-se, contudo, a continuidade de estudos de validação que corroborem com os atuais achados e os ampliem.

Palavras-chave: Motivação autodeterminada. Professores. Escala. Validação.
\end{abstract}

DOI: $10.1590 / 0104-4060.47470$

* O artigo resulta da pesquisa financiada pela Fundação de Amparo à Pesquisa do Rio Grande do Sul (FAPERGS) e pela Coordenação de Aperfeiçoamento de Pessoal de Nível Superior (CAPES).

** Pontifícia Universidade Católica do Rio Grande do Sul. Escola de Humanidades. Porto Alegre, Rio Grande do Sul. Av. Ipiranga, nº 6.681. Ipiranga. CEP: 90619-900. E-mail: tarciad@ gmail.com; bettina@pucrs.br 


\begin{abstract}
This study's main objective was to analyze psychometric evidence of validity and reliability of a Teachers Motivation Scale (EMD, Escala de Motivação Docente), in order to explore self-determined motivation exhibited by teachers. This scale was constructed in advance by the research group, and was developed based on the Self-Determination Theory (SDT), which approaches self-determined motivation as an intrinsic process, influenced by social context, taking into account basic psychological needs such as autonomy, competence and sense of belonging. After semantic and content analysis of all scale's items and once a pilot test was performed, a preliminary version of the EMD scale was applied to 398 Brazilian faculty members. These exploratory results led to the refinement of the scale. The prototype version of this EMD is now composed of twenty-four Likert items and embraces five dimensions: Performance, Personal Development, Teaching Practice, Continued Education and Institutional Insertion. The factorial analysis showed good internal consistency, confirming the proposed structural model. Although preliminary, the current results provide consistent evidence for the validity of the EMD construct and seem promising for studies related to teachers' motivational evaluation. It is recommended, however, that other validity studies which corroborate and amplify current findings be pursued.
\end{abstract}

Keywords: Self-determined motivation. Faculty members. Scale. Validation.

\title{
Introdução
}

O sucesso das políticas públicas e educacionais que almejam a qualidade da Educação Superior (BRASIL, 2014; MOROSINI et al., 2016) demanda por professores motivados. Teorias contemporâneas que tratam sobre a motivação afirmam que a autodeterminada (ou autônoma) é a que melhor representa o potencial humano naturalmente voltado ao crescimento e à integração intrapsíquica e social (DECI; RYAN, 2000), sendo bastante desejável a sua presença nos contextos educativos.

A motivação autodeterminada, como um conceito específico, tem sido estudada de modo sistemático pela Self-Determination Theory (SDT) (DECI; RYAN, 1985, 2000, 2002, 2008). De acordo com a SDT, a motivação autodeterminada possui locus de causalidade intrínseco ao self, levando a pessoa a manter alto nível de reflexão e consciência sobre suas escolhas e ações, desencadeando um processo contínuo de aprender mais sobre si mesma. Estudos apontam 
que a motivação autodeterminada em relação à docência está positivamente relacionada aos sentimentos de realização pessoal e negativamente associada à falta de energia, cansaço e esgotamento nas atividades docentes (FRIEDMAN; FARBER, 1992; PELLETIER et al., 2002; ROTH et al., 2007). Um professor intrinsecamente motivado tende a explorar ativamente as próprias capacidades, engajando-se e permanecendo espontaneamente envolvido em atividades percebidas como interessantes e recompensadoras em si mesmas, enfrentando desafios e desenvolvendo habilidades e competências necessárias à sua atuação. Além disso, a literatura sustenta, empiricamente, que a motivação autodeterminada dos professores para o ensino também promove a autonomia dos estudantes para a aprendizagem, como demonstra, por exemplo, um estudo com 132 professoras israelenses e seus 1.255 alunos de classe baixa e média, do $3^{\circ}$ ao $6^{\circ}$ ano, distribuídos em 62 turmas (de 6 a 12 em cada escola) (ROTH et al., 2007).

A SDT, em sua metateoria, assume que a motivação autodeterminada pode ser apoiada ou contrariada por influências sociocontextuais que se processam através das necessidades psicológicas de autonomia, competência e pertencimento/afiliação. Quando essas três necessidades são atendidas, conjunta e continuamente nas interações com o ambiente, tendem a produzir "nutrientes" que fortalecem o desenvolvimento e integração do self, promovendo vitalidade e bem-estar. São, portanto, necessidades associadas ao desenvolvimento psicossocial e à saúde psicológica, consideradas inatas e universais em sua essência, variando, sobretudo, no modo como são implementadas com respeito ao funcionamento real de cada pessoa nos diferentes contextos em que se insere (DECI; RYAN, 1985; RYAN; DECI, 2000).

Do ponto de vista conceitual e universal proposto pela SDT (RYAN; DECI, 2000; DECI; RYAN, 2008), o senso de autonomia é decorrente da percepção que a pessoa tem que suas ações e escolhas estão pautadas na própria vontade e no interesse genuíno sustentado pela coesão da atividade/comportamento com os valores pessoais, sendo relativamente livre de coerção ou pressões externas ao self. A autonomia se fundamenta, portanto, no livre arbítrio para originar um comportamento valorizado ou desejado, pressupondo autoconhecimento e responsabilização, sem, contudo, desconsiderar as circunstâncias externas e consequências reais das escolhas. A competência refere-se à percepção de ser capaz de produzir resultados desejáveis e evitar os indesejáveis, evidenciando a iniciativa e a crença na própria capacidade de realização, de aprendizagem e de habilidades sociais, voltadas à execução de atividades ou tarefas em diferentes níveis de dificuldade. Já o pertencimento ou a afiliação decorre da percepção do real interesse das outras pessoas na sua presença e existência, resultante da experiência prazerosa de interagir e compartilhar com os demais, da valorização 
da interação interpessoal e social e do genuíno apoio e crescimento oriundos das relações afetivas e socializadoras.

A motivação autodeterminada dos professores pode, portanto, ser afetada tanto por aspectos pessoais (crenças, expectativas, autoconhecimento) quanto contextuais, tais como: pressões institucionais, valorização e apoio social, funcionamento grupal (ROTH et al., 2007). Reafirma-se, assim, como um fenômeno complexo, multideterminado e multifacetado, que envolve a interação dinâmica e permanente de forças internas ao self, e contextuais, em direção à potencial integração e ao crescimento.

Apesar da relevância da motivação docente, sua produção científica ainda é insuficiente, e até incipiente, como aponta recente estudo bibliométrico realizado por Viseu et al. (2015), sendo quatro vezes menor do que a voltada para a motivação de estudantes e ainda mais escassa na América Latina. Muitos estudos focados na motivação do estudante, de diferentes níveis de ensino, trazem importantes contribuições (ARAÚJO; SILVA; FRANCO, 2014; CASTRO-CARRASCO et al., 2012; SOUZA, 2010;); mas evidenciam o docente como agente e não como sujeito do processo motivacional. Uma busca ${ }^{1}$ realizada pelos autores do presente estudo, nas bases Scientific Electronic Library Online - Scielo Brasil e Bibliografia Brasileira de Educação (BBE) - Inep, incluindo o período de 2000 a 2015, confirmou a escassez de publicações em língua portuguesa sobre a motivação docente, bem como a baixa indexação desses estudos em periódicos relevantes. Além disso, constatou-se que os estudos brasileiros que envolvem a motivação docente, em sua maioria, exploram primariamente construtos correlatos, como a satisfação no trabalho, as implicações sobre a aprendizagem, o estresse ou a saúde ocupacional, sendo a motivação observada mais como um fator coadjuvante.

Em parte, essa escassez de estudos pode estar associada ao fato de a motivação ser, sob a ótica da pesquisa científica, um construto não diretamente observável, sendo passível de identificação apenas por meio de suas manifestações e expressões indiretas (os traços latentes). A investigação em larga escala de construtos psicológicos dessa natureza, visando à sistematização do conhecimento empírico e à replicabilidade dos estudos, retroalimentando o conhecimento teórico existente, demanda pela utilização de instrumentos ou de técnicas que permitam acessar as variáveis latentes através dos elementos observáveis (comportamentos, atitudes, ações), pressupondo estudos quantita-

1 Os descritores utilizados foram motivação; processo(s) motivacional(is); motivação para ensinar; motivação escolar; motivação intrínseca; motivação extrínseca, isolados e em conjunto com educação superior; ensino superior; universidade; docente(s); professor(es). 
tivos que envolvem conhecimento psicométrico ${ }^{2}$. Nesse sentido, a utilização de instrumentos de pesquisa confiáveis e válidos, reconhecidos cientificamente, apoiados em uma métrica que permita conhecer a realidade motivacional dos professores, apontando fatores comuns e especificidades, são de suma importância.

A teoria das NPBs (Necessidades Psicológicas Básicas), incorporada à SDT, já originou diversos instrumentos para avaliar a motivação autodeterminada, na perspectiva da satisfação das necessidades, em contextos globais (GAGNÉ, 2003) e em contextos específicos, tais como: o trabalho (DECI et al., 2001; GAGNÉ et al., 2015), o exercício físico (VLANCHOPOULOS; MICHAILIDOUS, 2006) ou os relacionamentos (LA GUARDIA et al., 2000). Alguns, mais recentes, avaliam tanto a satisfação quanto a frustração das NPBs (CHEN et al., 2015). No entanto, a maioria desses instrumentos tem recebido críticas por não possuírem estudos suficientes que atestem sua validade psicométrica e/ou invariância transcultural (JOHNSTON; FINNEY, 2010). Além disso, não estão adaptados e validados para uso no Brasil, nem são específicos para avaliar a motivação docente.

Este artigo, portanto, apresenta uma nova medida denominada Escala de Motivação Docente (EMD), destinada a avaliar a motivação autodeterminada de professores a partir dos pressupostos da SDT. O estudo teve por objetivo desenvolver a escala e obter evidências de validade e confiabilidade, a partir dos dados oriundos de 398 professores universitários brasileiros. Inicialmente, descrevemos o processo de desenvolvimento da escala, especificamente a construção dos itens, a validação de conteúdo e o teste piloto. Em seguida, foram apresentadas evidências psicométricas com base na estrutura interna da EMD, que levaram ao refinamento da escala. A versão refinada da EMD, composta por 24 itens, foi analisada por meio de análise fatorial confirmatória, permitindo caracterizar a sua estrutura multifatorial composta por cinco dimensões que, em sua essência, incorporam as NPBs relacionadas à docência.

\section{Método}

\section{Desenvolvimento da EMD}

A EMD foi desenvolvida a partir do conhecimento sustentado em três pilares, além de seguir os protocolos recomendados pela literatura (PASQUA-

2 A Psicometria representa a teoria e a técnica de medida dos processos mentais, especialmente aplicada na área da Psicologia e da Educação, permitindo que os traços latentes de um construto psicológico, como a motivação, sejam representados por uma variável operacional, ou seja, um comportamento, ação ou atitude que o expresse com a maior exatidão e amplitude possível. 
LI, 2010). (1). Na literatura, por meio das teorias da motivação e do contexto contemporâneo da docência na Educação Superior, buscando compreender de modo mais específico as contribuições da SDT e as implicações das NPBs nos processos motivacionais imbricados na carreira docente. Compilou-se o estado da arte das pesquisas sobre o construto motivação, especialmente quando focado no contexto educacional e/ou profissional, confirmando a aplicabilidade da teoria e a existência de diversas medidas fundamentadas na SDT, porém, direcionadas a contextos diversos, ainda sem validação para o Brasil. (2). Em estudos empíricos qualitativos e exploratórios com professores universitários, realizados pela equipe de pesquisa, um deles (DAVOGLIO; SPAGNOLO; SANTOS, 2016, no prelo) objetivou explorar os indicadores motivacionais para a permanência na carreira docente; outro estudo (DAVOGLIO; SANTOS; TIMM; CONZATTI, 2017) visou a definição operacional das NPBs para professores universitários. (3). No know-how sobre processos motivacionais em contextos educativos, advindos da formação dos pesquisadores e da atuação do grupo de pesquisa Processos Motivacionais em Contextos Educativos (PROMOT) ${ }^{3}$ que desenvolve o estudo, em mais de uma década de pesquisas sobre motivação docente e discente.

Assim, inicialmente, foram desenvolvidas 43 afirmações para especificar aspectos motivacionais autodeterminados ou interferências sobre os mesmos, específicos para o docente, que evidenciassem, ao mesmo tempo, elementos pessoais e suporte sociocontextual relacionados às NPBs. A intenção era produzir itens que refletissem particularidades do contexto docente, com foco específico na Educação Superior, referenciadas nos estudos exploratórios e na literatura como elementos significativos à motivação para engajar-se na docência (tais como: relações interpessoais, formação continuada, gosto/prazer na prática docente, ambiente acadêmico, desafios e a inovação, compartilhamento do conhecimento, percepção de autonomia) e ao mesmo tempo focassem nas demandas individuais fomentadas pelas NPBs. Propusemos, assim, a exploração das NPBs inerentes à docência abordando-as conceitualmente como elementos pessoais e de autoconhecimento, contingenciais e políticos do cenário acadêmico/institucional.

A análise de validade de conteúdo dessa versão inicial incluiu a avaliação detalhada da escala por juízes peritos (cinco professores/pesquisadores com doutoramento em áreas da Educação e Psicologia, especialistas no estudo da motivação e/ ou dos contextos da educação, todos com experiência na utilização e/ou construção de instrumentos psicométricos). Todos os juízes preencheram um formulário de avaliação, tendo conhecimento das definições constitutivas das NPBs e pressupostos

3 Escola de Humanidades, Pontifícia Universidade Católica do Rio Grande do Sul (PUCRS). 
teóricos da SDT. Cada item foi discutido tendo em vista o potencial semântico e de compreensão, a redundância/repetição de termos e a representatividade teórica conceitual, além da adequação da estrutura gráfica da escala e da pontuação. Após a avaliação aprofundada desses resultados, optamos pela retirada de seis itens, primando pela manutenção daqueles com maior concordância entre os juízes e de alguns outros com concordância menos unânime, porém, com potencial para serem testados empiricamente, levando em conta a possibilidade de exclusão. Um teste piloto com 10 professores, com características similares à população alvo (atuavam na graduação e pós-graduação, oriundos de diferentes IES, regiões e áreas de conhecimento, masculinos e femininos, voluntariamente disponíveis para participar), foi conduzido para verificar as condições de aplicação (tempo, instruções, bem como a compreensão e clareza dos itens), gerando sugestões de ajustes idiomáticos pontuais, considerando-se assim a escala pronta para ser testada.

\section{Evidências da estrutura fatorial e consistência interna da EMD}

\section{Participantes}

Um total de 398 professores universitários (57\% mulheres), com idade média de 41,2 anos (DP = 9,7), atuando, prioritariamente, em instituições de Educação Superior privadas (90,1\%) nas regiões Sul $(38,2 \%)$ e Nordeste $(61,8 \%)$ do Brasil participaram deste estudo. O tamanho da amostra respeitou o critério amostra: item, considerado adequado para as análises pretendidas quando corresponde a pelo menos 10 participantes por item (HAIR et al., 2009).

\section{Instrumento}

Escala de Motivação Docente (EMD): Neste estudo os professores responderam à versão inicial da EMD, composta por 37 itens, de autorrelato. No entanto, o refinamento dessa versão, decorrente das análises psicométricas realizadas, originou uma versão prototípica de 24 itens, os quais compõem as cinco dimensões da escala. O conteúdo dos itens explora a valorização da qualidade autônoma ou autodeterminada da motivação, a qual se organiza a partir das NPBs de autonomia, competência e afiliação/pertencimento, tendo como base teórica a SDT. Cada item foi avaliado por meio de uma escala do tipo Likert de cinco pontos $(1=$ nada aplicável; $5=$ totalmente aplicável $)$, variando de acordo com o grau de aplicação dos comportamentos e crenças descritos na sentença às vivências do participante em relação à docência. No entanto, sete itens $(6,8,12,16,17,18,19)$ devem ser computados com escores inversos (por 
exemplo, $1=5$ ), a fim de permitir a comparação com os resultados dos demais itens, a partir de uma mesma lógica de qualidade motivacional. Ou seja, quanto maior a pontuação mais próximo da motivação autodeterminada ou autônoma.

\section{Procedimentos de coleta e análise de dados}

Os dados foram coletados, em versão impressa, a partir do contato direto e/ou institucional com os professores, os quais foram convidados a participar recebendo informações detalhadas sobre os objetivos da pesquisa e sua implicação. Aqueles que aceitaram participar, de forma voluntária, assinaram o termo de consentimento livre e esclarecido, tendo assegurado o direito ao anonimato e confidencialidade de suas informações pessoais. O projeto ${ }^{4}$ foi aprovado pelo Comitê de Ética em Pesquisa (Protocolo CEP/PUCRS nº 10/05132).

As variáveis foram submetidas a análises fatoriais, que consistem em um conjunto de técnicas estatísticas fundamentais para explorar a validade de construto de um instrumento de medida (BROWN, 2006; HAIR et al., 2009; PACICO; HUTZ, 2015), podendo ser do tipo exploratória ou confirmatória. A análise fatorial exploratória (AFE) permitiu uma verificação preliminar do número de fatores e padrões de cargas gerados espontaneamente pelos dados e da sua adequação para as análises pretendidas. A AFE não tem como finalidade testar uma teoria, por isso esse passo não é necessariamente uma exigência na validação de um modelo estrutural teórico (HAIR et al., 2009), porém, é sempre recomendado realizá-las, especialmente quando o modelo estrutural é incipiente e ainda não consolidado por estudos empíricos (HU; BENTLER, 1999). Quando realizadas com os mesmos dados que fornecerão resultados confirmatórios, são essenciais para verificar a presença de problemas metodológicos nas análises ou no conjunto de dados examinados (tais como: adequação amostral, dimensionalidade, cargas cruzadas, fatores com poucos itens, itens com pouca coerência) (HU; BENTLER, 1999).

Os fatores foram extraídos pelo método de componentes principais e rotação oblíqua (promax), pressupondo correlação entre as variáveis. A adequação dos dados para as análises foi dada pelo teste Kaiser-Meyer-Olklin (KMO), considerando melhores resultados os mais próximos a $1(>0,90=$ excelente; entre 0,80 a $0,89=$ bom) e pelo teste de Esfericidade de Bartlett estatisticamente

4 O projeto que abarca a pesquisa contou com o financiamento da Fundação de Amparo à Pesquisa do Rio Grande do Sul (FAPERGS), Coordenação de Aperfeiçoamento de Pessoal de Nível Superior (Capes) e Conselho Nacional de Desenvolvimento Científico e Tecnológico (CNPq). 
significante $(\mathrm{p}<0,05)$. A definição do número de fatores retidos baseou-se no teste Scree e no critério de autovalor de Kaiser ( $>1)$, considerado adequado para 20 a 50 variáveis (TABACHNICK; FIDELL, 2007).

A análise fatorial confirmatória (AFCF), por outro lado, serve para predizer o quanto os dados reais se ajustam ao modelo hipotetizado, o qual deve ser especificado a priori com base na teoria, sendo, neste estudo, composto por cinco dimensões, correlacionadas entre si. Essa adequação e qualidade do ajuste do modelo aos dados foi estimada por meio dos índices de ajuste absoluto: raiz quadrada média residual (SRMR) e raiz quadrada média do erro de aproximação (RMSEA), em que valores $\leq 0,08 \mathrm{e} \leq 0,06$, respectivamente, indicam ajustes aceitáveis para o modelo (BROWN, 2006; HAIR et al., 2009). Outros dois índices, de ajuste relativo (TLI; Tucker-Lewis Index) e de ajuste comparativo (CFI), foram examinados, sendo valores entre 0,90 e 0,95 indicativos de bom ajuste (BROWN, 2006). Além disso, o qui-quadrado sobre graus de liberdade $\left(\mathrm{X}^{2} / \mathrm{gl}=\mathrm{df}\right)$ permitiu obter mais um indicador de ajuste do modelo, para o qual valores $<3$ são preferíveis, mas valores $<5$ são tolerados (HAIR et al., 2009).

A confiabilidade (ou fidedignidade) da escala foi verificada pelo coeficiente alfa, comumente indicado para itens politômicos, considerando que quanto maior a correlação média entre os itens maior será o valor de alfa (pode variar de $0 \mathrm{a} 1$ ), o qual avalia o grau em que os itens da matriz de dados estão correlacionados, o que se reflete em maior consistência interna (HAIR et al., 2009). Os fatores ou dimensões extraídas foram nomeadas e interpretadas com base na SDT e nas especificidades do contexto docente. As análises descritivas e AFE foram realizadas no SPSS, versão 17.0, e a AFCF utilizou o software Mplus, versão 6.1.

\section{Resultados e discussão}

A AFCF inicial no conjunto de 37 itens evidenciou ajustes insatisfatórios para o modelo estrutural hipotetizado em todos os índices (RMSEA $=0,097$; $\mathrm{CFI}=0,651 ; \mathrm{TLI}=0,602 ; \mathrm{SRMR}=0,094)$. A AFE com esse conjunto de itens evidenciou a natureza multifatorial dos dados, porém, confirmou as inconsistências, revelando itens com cargas fatoriais abaixo do esperado $(<0,40)$, cargas cruzadas nas dimensões, além de dimensões compostas por poucos itens. Tais evidências levaram ao refinamento da escala, com a retenção de 24 itens, submetidos a nova AFE, corroborando a ideia inicial de que talvez nem todos os itens fossem viáveis. A medida Kaiser-Meyer-Olkin $(\mathrm{KMO}=0,84) \mathrm{e}$ a significância para as correlações (teste de esfericidade de Bartlett, $p=0,000$ ) apontou a adequação dos dados para as análises fatoriais. O modelo exploratório 
extraído, com rotação oblíqua, revelou a presença de 5 dimensões pelo critério Kaiser (autovalor > 1), explicando 49,3\% da variância total, considerada satisfatória diante da adoção da perspectiva confirmatória para o estabelecimento do modelo estrutural.

Essa estrutura fatorial simples, composta por 5 dimensões, evidenciou cargas fatoriais exploratórias significativas, ou seja, $\geq 0,45$ para todos os itens (Tabela 1), o que a literatura considera razoável $(>0,45)$ a excelente $(>0,71)$ (COMREY; LEE, 1992). A consistência interna (alfa) de todas as dimensões ficou menor do que a obtida para o total de itens (Tabela 1), porém, um valor "entre 0,60 e 0,70 pode ser aceitável desde que outros indicadores de validade de construto do modelo sejam bons" (HAIR et al., 2009, p. 592). Note-se que o número de itens que compõe o fator pode influenciar o valor de alfa, ou seja, fatores com mais itens tendem a ter valor de alfa mais elevado (HAIR et al., 2009).

\section{TABELA 1 - CARGAS FATORIAIS E COEFICIENTE ALFA PARAAS DIMEN- SÕES DA ESCALA DE MOTIVAÇÃO DOCENTE}

\begin{tabular}{|c|c|c|c|c|c|}
\hline \multirow[t]{2}{*}{ Itens da EMD } & \multicolumn{5}{|c|}{ Dimensões ou fatores } \\
\hline & D1 & D2 & D3 & D4 & D5 \\
\hline Item 18 & 068 & & & & \\
\hline Item 8 & 0,67 & & & & \\
\hline Item 6 & 0,63 & & & & \\
\hline Item 12 & 0,61 & & & & \\
\hline Item 19 & 0,60 & & & & \\
\hline Item 16 & 0,57 & & & & \\
\hline Item 17 & 0,50 & & & & \\
\hline Item 11 & & 0,66 & & & \\
\hline Item 14 & & 0,63 & & & \\
\hline Item 1 & & 0,59 & & & \\
\hline Item 9 & & 0,54 & & & \\
\hline Item 10 & & 0,55 & & & \\
\hline Item 15 & & 0,45 & & & \\
\hline Item 24 & & 0,45 & & & \\
\hline Item 21 & & & 0,78 & & \\
\hline Item 22 & & & 0,78 & & \\
\hline Item 23 & & & 0,66 & & \\
\hline Item 13 & & & 0,57 & & \\
\hline Item 4 & & & & 0,78 & \\
\hline Item 7 & & & & 0,71 & \\
\hline Item 5 & & & & 0,67 & \\
\hline Item 2 & & & & & 0,73 \\
\hline Item 3 & & & & & 0,63 \\
\hline Item 20 & & & & & 0,59 \\
\hline Alfa Confiabilidade & 0,721 & 0,741 & 0,742 & 0,674 & 0,620 \\
\hline
\end{tabular}

FONTE: Elaborada pelas autoras (2016). 
O sentido teórico dos itens consiste no principal critério para estabelecer as dimensões latentes de um instrumento, por isso, a AFCF exige um modelo estrutural definido a priori, ao qual os dados reais se ajustem satisfatoriamente. Com base no modelo teórico previamente definido e testado com AFE (como mostra a Tabela 1), os resultados da AFCF evidenciaram que esse modelo estrutural de 5 dimensões para o total dos 24 itens foi estatisticamente adequado, apresentando os seguintes índices de qualidade de ajuste: $\mathrm{X}^{2} / \mathrm{df}=2,08$; RMSEA $(90 \%$ IC $)=0,052(0,046-0,059) ; \mathrm{CFI}=0,942 ; \mathrm{TLI}=0,934 ; \mathrm{SRMR}=0,061$, todos dentro dos parâmetros recomendados pela literatura. Além desses índices, na adequação do modelo confirmatório, observamos, como mostra o diagrama de caminho (Figura 1), que as cargas padronizadas (não submetidas aos efeitos das diferenças entre correlações e covariâncias) foram significativas $(\mathrm{p}<0,001)$ e dentro dos parâmetros aceitáveis (> 0,50) (HAIR et al., 2009), com exceção apenas do item $17(0,45)$. A carga fatorial desse item tanto pode refletir uma idiossincrasia amostral quanto uma limitação efetiva da variável, o que deve ser depurado na continuidade do processo de validação da escala.

Por outro lado, as correlações fatoriais resultantes da AFCE foram expressivas entre todas as dimensões, variando de 0,42 a 0,81 (D2<->D $3=0,81$; D2 <$->$ D4 $=0,74 ;$ D2 <->D5=0,61; D3<->D4=0,58; D3<->D5=0,42; D4<->D5=0,55), com exceção da dimensão $1(\mathrm{D} 1<->\mathrm{D} 2=0,32 ; \mathrm{D} 1<->\mathrm{D} 3=0,14 ; \mathrm{D} 1<->\mathrm{D} 4=0,22$; $\mathrm{D} 1<->\mathrm{D} 5=0,22)$. É relevante destacar que, em pesquisa aplicada, quando a correlação fatorial excede a 0,85 , pode ser considerada um critério sugestivo de validade discriminante deficiente entre os fatores (BROWN, 2006). Portanto, é possível inferir que a estrutura proposta para a EMD não possui construtos fatoriais sobrepostos.

Do ponto de vista metodológico, somente um modelo estrutural que satisfaz critérios teóricos e estatísticos evidencia validade de construto e é passível de interpretabilidade. Diante disso, as cinco dimensões que compõem o modelo estrutural da EMD, especificadas no Quadro 1, ao atenderem tais critérios, mostraram-se representativas da motivação docente, na perspectiva da autodeterminação. 


\section{FIGURA 1 - MODELO ESTRUTURAL DA ESCALA DE MOTIVAÇÃO DO- CENTE}

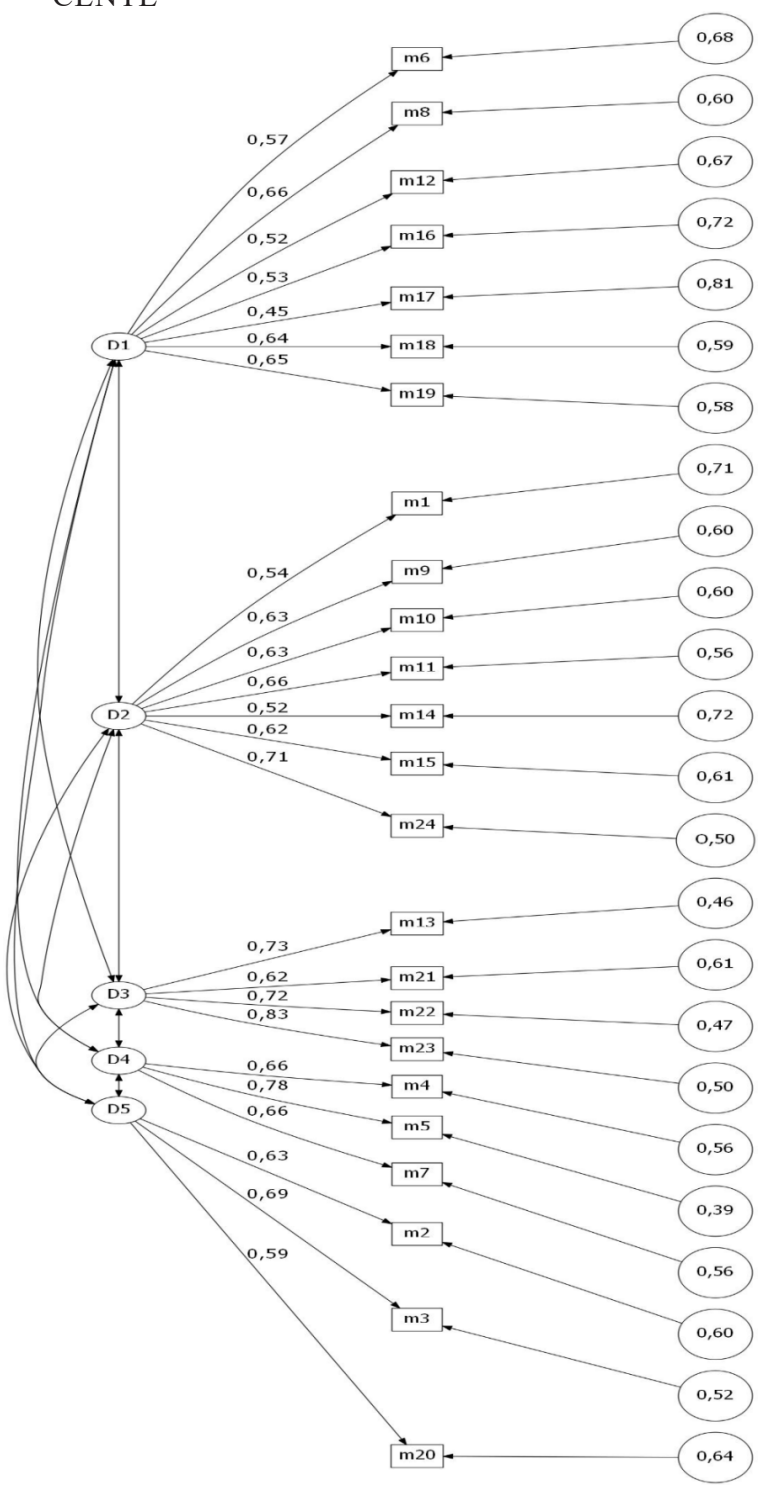

Nota: Elipses $=$ fatores latentes; retângulos = variáveis observadas; círculos $=$ os erros residuais . FONTE: Elaborado pelas autoras (2016). 


\section{QUADRO 1 - DIMENSÕES E ITENS DA ESCALA DE MOTIVAÇÃO DOCENTE}

\begin{tabular}{|c|c|}
\hline Dimensões (D) & Descrição dos itens \\
\hline \multirow{6}{*}{$\begin{array}{l}\text { PERFORMANCE (D1): Foca-se em situa- } \\
\text { ções de pressão e expectativa que afetam a } \\
\text { qualidade do desempenho e a percepção de } \\
\text { capacidades, as quais possuem potencial ne- } \\
\text { gativo sobre a motivação autodeterminada } \\
\text { por serem antagônicas com as tendências à } \\
\text { integração e coesão psicológica. Por isso, } \\
\text { os escores desses itens foram codificados } \\
\text { de modo inverso, pois, atuam no sentido de } \\
\text { minar a autodeterminação.É composta pelos } \\
\text { itens } 6,8,12,16,17,18,19 \text {. }\end{array}$} & $\begin{array}{l}\text { Sinto-me menos capaz quando preciso lidar com } \\
\text { conteúdos novos. }\end{array}$ \\
\hline & $\begin{array}{l}\text { A falta de apoio dos pares limita minha disposição para } \\
\text { enfrentar as dificuldades ou oportunidades docentes. }\end{array}$ \\
\hline & $\begin{array}{l}\text { A remuneração pelo trabalho docente interfere na } \\
\text { percepção das capacidades que possuo. }\end{array}$ \\
\hline & $\begin{array}{l}\text { Sinto que é difícil trabalhar cooperativamente no meio } \\
\text { docente. }\end{array}$ \\
\hline & $\begin{array}{l}\text { A maioria das vezes preciso do reconhecimento dos } \\
\text { colegas e da instituição para me sentir competente. }\end{array}$ \\
\hline & $\begin{array}{l}\text { Às vezes deixo de fazer algo do meu interesse pelo } \\
\text { receio me sentir constrangido(a). }\end{array}$ \\
\hline \multirow{7}{*}{$\begin{array}{l}\text { DESENVOLVIMENTO (D2): Refere-se } \\
\text { às atitudes e escolhas do docente que estão } \\
\text { voltadas ao crescimento e desenvolvimento, } \\
\text { as quais se mostram coesas e integradas ao } \\
\text { self. Alinha-se a um processo contínuo de } \\
\text { aprender mais sobre si mesmo e na intera- } \\
\text { ção com os demais, posicionando a pessoa } \\
\text { na origem das próprias ações, evidenciando } \\
\text { reflexão e consciência sobre escolhas e pro- } \\
\text { pósitos. É constituída pelos itens } 1,9,10,11 \text {, } \\
14,15,24 \text {. }\end{array}$} & $\begin{array}{l}\text { Compartilhar ideias, sentimentos e dúvidas com meus } \\
\text { colegas influencia positivamente meu trabalho. }\end{array}$ \\
\hline & $\begin{array}{l}\text { Continuo estudando porque acho que ainda tenho muito } \\
\text { a aprender. }\end{array}$ \\
\hline & Sempre avalio criticamente minhas ações docentes. \\
\hline & $\begin{array}{l}\text { Interajo e socializo com meus colegas como forma de } \\
\text { desenvolvimento. }\end{array}$ \\
\hline & $\begin{array}{l}\text { Na minha prática, tento levar os alunos a compreender } \\
\text { que errar faz parte da aprendizagem. }\end{array}$ \\
\hline & $\begin{array}{l}\text { Invisto na profissão docente porque me proporciona } \\
\text { realização de meus objetivos. }\end{array}$ \\
\hline & $\begin{array}{l}\text { Gosto de trabalhar cooperativamente, mesmo com } \\
\text { colegas que não conheço. }\end{array}$ \\
\hline \multirow{4}{*}{$\begin{array}{l}\text { PRÁTICA DOCENTE (D3): Composta } \\
\text { por quatro itens }(13,21,22,23) \text {, foca-se no } \\
\text { alinhamento entre os interesses pessoais e o } \\
\text { fazer docente, destacando a harmonia entre a } \\
\text { vontade pessoal e as demandas profissionais. }\end{array}$} & $\begin{array}{l}\text { Sinto-me bem quando posso arriscar-me com soluções } \\
\text { criativas. }\end{array}$ \\
\hline & Valorizo ter atividades e horários de trabalho flexíveis. \\
\hline & $\begin{array}{l}\text { Esforço-me para que meus alunos e colegas sintam que } \\
\text { me importo com eles. }\end{array}$ \\
\hline & \\
\hline \multirow{3}{*}{$\begin{array}{l}\text { FORMAÇÃO CONTINUADA (D4): Re- } \\
\text { fere-se à busca por qualificação e desafios } \\
\text { como compromisso pessoal do docente. A } \\
\text { motivação autodeterminada tende a ser ca- } \\
\text { racterística de pessoas que possuem iniciati- } \\
\text { va e crença na própria capacidade de realiza- } \\
\text { ção. Inclui os itens }(4,5,7) \text {. }\end{array}$} & Realizo cursos de atualizaç \\
\hline & $\begin{array}{l}\text { Gosto quando a instituição me oferece oportunidades } \\
\text { para realizar atividades desafiadoras. }\end{array}$ \\
\hline & $\begin{array}{l}\text { Participo de atividades de educação continuada porque } \\
\text { quero. }\end{array}$ \\
\hline \multirow{3}{*}{$\begin{array}{l}\text { INSERÇÃO INSTITUCIONAL (D5): } \\
\text { Formada por três itens }(2,3,20) \text { explora a vi- } \\
\text { vência de integração do docente no ambien- } \\
\text { te institucional. As experiências de escolher, } \\
\text { interagir e compartilhar, promovidas pelo } \\
\text { ambiente, funcionam como estímulos para } \\
\text { o engajamento e a responsabilização que } \\
\text { acompanham as ações autodeterminadas. }\end{array}$} & $\begin{array}{l}\text { Sinto que tenho liberdade para negociar a participação } \\
\text { nas diferentes atividades docentes. }\end{array}$ \\
\hline & $\begin{array}{l}\text { Os espaços de cooperação entre os docentes } \\
\text { promovidos pela instituição são fonte de prazer para } \\
\text { mim. }\end{array}$ \\
\hline & $\begin{array}{l}\text { Sinto-me livre para expressar meus sentimentos e } \\
\text { ideias no trabalho. }\end{array}$ \\
\hline
\end{tabular}

FONTE: Elaborada pelas autoras com base nos resultados do presente estudo. 
Em conjunto, essas cinco dimensões apontam que a possibilidade de livre escolha da forma e ritmo de realização de uma atividade, a capacitação e domínio dessa atividade e o reconhecimento de sentimentos e apoio por pessoas significativas, como colegas e gestores, podem aumentar a autodeterminação para ações docentes. Contrariamente, a percepção de controle ou ausência de aprovação, a excessiva preocupação com as expectativas, ou o desempenho, tende a interferir na qualidade autônoma da motivação, e, por conseguinte, na intenção de prosseguir livremente naquela direção, devendo, porém, todas as dimensões serem analisadas em conjunto para validar sua interpretabilidade.

Se a motivação autodeterminada pode ser mensurada a partir de suas manifestações, então seria possível identificar pessoas e contextos que necessitam de apoio ou intervenção para melhor desenvolvê-la ou mantê-la, elevando o nível de autoconhecimento e conhecimento da realidade imediata que cerca a atuação do docente na Educação Superior, condizente com a aplicabilidade da EMD. Quando há autodeterminação em relação à docência, o professor experimenta sensação de liberdade e engajamento para fazer o que considera interessante, pessoalmente importante e vitalizante, cumprindo metas, sendo criativo e preservando a disposição e a saúde psicológica.

Quanto aos índices de modificação gerados pelo software estatístico (indicam o quanto o qui-quadrado do modelo geral seria reduzido e o ajuste melhorado se houvesse liberação de caminho), neste momento, não foram considerados, entendendo-se que mudanças no modelo não devem ser baseadas apenas nesses índices, especialmente, diante da incipiência da aplicação empírica da escala e a evidência de bons índices de qualidade do ajuste do modelo estrutural (HAIR et al., 2009).

\section{Considerações finais}

Os resultados psicométricos apresentados para a versão prototípica de 24 itens da EMD demonstraram o potencial de validade e de confiabilidade da escala para identificar aspectos relevantes à motivação autodeterminada implícitos na docência. $\mathrm{O}$ estudo forneceu evidências da natureza multifatorial da escala, composta por cinco dimensões latentes, que consideram as demandas e correlações implicadas nas NPBs, abordando-as como resultado da transação bem-sucedida entre o indivíduo ativo e singular e o contexto interpessoal e institucional, em diferentes perspectivas em relação à docência. 
O modelo estrutural da EMD satisfez diversas diretrizes relacionadas ao processo de validação, entre as quais, além dos índices de ajuste da qualidade adequados, a exigência de que o modelo congênere apresente fatores comuns compostos pelo menos por três variáveis indicadoras significativas (HAIR et al., 2009), o que foi contemplado para todas as dimensões da EMD. No entanto, a fim de obter maior equilíbrio entre a representatividade dos itens nas dimensões, consideramos a possibilidade de testar em estudos futuros a introdução de alguns novos itens nas dimensões D3, D4, D5, alinhados a essas e respaldados pela teoria, o que poderia também resultar em maior consistência interna das dimensões.

A utilização de amostra heterogênea para técnicas de análise fatorial é um aspecto desejado, pois possibilita representatividade mais ampla da população alvo e variância que evidencie as correlações possíveis entre os dados reais e o modelo estrutural. Contudo, recomendamos que esse modelo estrutural seja testado em amostras homogêneas (por exemplo, professores oriundos de uma mesma região), considerando, além da variação cultural existente no país, a diversidade de políticas docentes submetidas a normatizações locais/estaduais a fim de averiguar a especificidade/sensibilidade do modelo. Além disso, a comparação entre amostras oriundas de instituições públicas e privadas pode também trazer acréscimos à validade de conteúdo.

Por fim, entendemos que a validação de uma medida é um processo permanente, em construção contínua por meio de novas evidências que irão sendo incorporadas ao conhecimento já consolidado, gerando uma retroalimentação entre a teoria e a realidade que tornam a medida cada vez mais válida e confiável, o que é altamente desejável quando é abordado um construto complexo como a motivação. Nessa lógica, as evidências de validade e confiabilidade da EMD apresentadas devem ser compreendidas como preliminares e demandantes por novos estudos que corroborem os achados e os ampliem, por exemplo, testando a validade convergente e discriminante, ou contextos transculturais.

\section{REFERÊNCIAS}

ARAUJO, M. V.; SILVA, J.W. B.; FRANCO, E. M. Motivação para o aprendizado em estudantes de graduação em Psicologia. Psicologia teoria e prática, São Paulo, v. 16, n. 2, p. 185-198, ago. 2014. Disponível em: <http://pepsic.bvsalud.org/scielo. php?script=sci_arttext\&pid=S1516-36872014000200016\&lng $=$ pt\&nrm=iso $>$. Acesso em: 10 jan. 2016. 
BRASIL. Planejando a próxima década: conhecendo as 20 metas do Plano Nacional de Educação. Ministério da Educação - Secretaria de Articulação com os Sistemas de Ensino (MEC/SASE), 2014. Disponível em: <http://pne.mec.gov.br/images/pdf/pne_conhecendo_20_metas.pdf $>$. Acesso em: 19 mar. 2015.

BROWN, T. A. Confirmatory Factor Analysis for Applied Research. New York: Guilford Press, 2006.

CASTRO-CARRASCO, P. J. et al. Teorias subjetivas de professores sobre a motivação e suas expectativas de sucesso e fracasso escolar. Educar em Revista, n. 46, p. 159-172, 2012. Disponível em: <http://dx.doi.org/10.1590/S0104-40602012000400012>. Acesso em: 07 ago. 2015.

CHEN, B. et al. Basic psychological need satisfaction, need frustration, and need strength across four cultures. Motivation and Emotion, v. 39, n. 2, p. 216-236, 2015.

COMREY, A. L.; LEE, H. B. A first course in factor analysis. 2. ed. Hillsdale, New Jersey: Erlbaum, 1992.

DAVOGLIO, T. R.; SPAGNOLO, C.; SANTOS, B. S. Motivação para a permanência na profissão: a percepção dos docentes universitários. Psicologia Escolar e Educacional. 2016. No prelo.

DAVOGLIO, T. R.; SANTOS, B. S.; TIMM, J. W.; CONZATTI, F. B. K. Necessidades Psicológicas Básicas: definições operacionais na docência universitária. ETD - Educação Temática Digital, Campinas, SP, v. 19, n. 2, p. 510-531, abr./jun. 2017. doi: https://doi. org/10.20396/etd.v19i2.8644789

DECI, E. L.; RYAN, R. M. Intrinsic motivation and self-determination in human behavior. New York: Plenum Press, 1985.

DECI, E. L.; RYAN, R. M. The "what" and "why" of goal pursuits: human needs and self-determination of behavior. Psychological Inquiry, v. 11, n. 4, 2000. Disponível em: $<$ http://www.tandfonline.com/doi/abs/10.1207/S15327965PLI1104_01>. Acesso em: 17 mar. 2015.

DECI, E. L.; RYAN, R. M. Handbook of Self-determination Research. Rochester, N.Y.: The University of Rochester Press, 2002.

DECI, E. L.; RYAN, R. M. Facilitating optimal motivation and psychological well-being across life's domains. Canadian Psychological Association, v. 49, n. 1, p. 14-23, 2008.

DECI, E. L. et al. Need satisfaction, motivation, and well-being in the work organizations of a former eastern bloc country: a cross-cultural study of self-determination. Personality and Social Psychology, v. 27, n. 8, p. 930-942, ago. 2001.

FRIEDMAN, I. A.; FARBER, B. A. Professional self-concept as a predictor of teacher burnout. Journal of Educational Research, v. 86, n. 1, p. 28-35, set./out. 1992.

GAGNÉ, M. The role of autonomy support and autonomy orientation in prosocial behavior engagement. Motivation and Emotion, v. 27, n. 3, p. 199-223, set. 2003. 
GAGNÉ, M. et al. The Multidimensional work motivation scale: validation evidence in seven languages and nine countries. European Journal of Work and Organizational Psychology, v. 24, n. 2, p. 178-196, 2015.

HAIR, J. F. et al. Análise multivariada de dados. 6. ed. Porto Alegre: Bookman, 2009.

HU, L.; BENTLER, P. M. Cutoff criteria for fit indexes in covariance structure analysis: conventional criteria versus new alternatives. Structural Equation Modeling: A Multidisciplinar Jornal, v. 6, n. 1, p. 1-55, 1999.

JOHNSTON, M.; FINNEY, S. J. Measuring basic needs satisfaction: evaluating previous research and conducting new psychometric evaluations of the Basic Needs Satisfaction in General Scale. Contemporary Educational Psychology, v. 35, n. 4, p. 280-296, 2010.

LA GUARDIA et al. Within-person variation in security of attachment: a self-determination theory perspective on attachment, need fulfillment, and well-being. Journal of Personality and Social Psychology, v. 79, n.3, p. 367-384, set. 2000.

MOROSINI, M. C. et al. Quality of higher education and the complex exercise of proposing indicators. Rev. Bras. Educ., Rio de Janeiro, v. 21, n. 64, mar. 2016. Disponível em: <http://dx.doi.org/10.1590/S1413-24782016216402>. Acesso em: 03 maio 2016.

PACICO, J. C.; HUTZ, C. S. Validade. In: HUTZ, C. S.; BANDEIRA, D. R.; TRENTINI, C. M. Psicometria. Porto Alegre: Artmed, 2015. p. 71-84.

PASQUALI, L. Escalas Psicométricas. In: PASQUALI, L. et al. Instrumentação psicológica: fundamentos e práticas. Porto Alegre: Artmed, 2010. p. 116-135.

PELLETIER, L. G.; SGUIN-LEVESQUE, C.; LEGAULT, L. Pressure from above and pressure from below as determinants of teachers' motivation and teaching behaviors. Journal of Educational Psychology, v. 94, n. 1, mar. 2002. Disponível em: $<$ http:// psycnet.apa.org/?\&fa=main.doiLanding \&doi=10.1037/0022-0663.94.1.186>. Acesso em: 15 abr. 2015.

RYAN, R. M.; DECI, E. L. Self-determination theory and the facilitation of intrinsic motivation, social development, and well-being. American Psychologist, v. 55, n. 1, p. 68-78, jan. 2000. Disponível em: <https://selfdeterminationtheory.org/SDT/documents/2000_RyanDeci_SDT.pdf $>$. Acesso em: 25 abr. 2015.

ROTH, G. et al. Autonomous motivation for teaching: how self-determined teaching may lead to self-determined learning. Journal of Educational Psychology, v. 99, n. 4, p. 761-774, nov. 2007.

SOUZA, L. F. N. I. de. Estratégias de aprendizagem e fatores motivacionais relacionados. Educar em Revista, Curitiba, n. 36, p. 95-107, 2010. Disponível em: <http://www.scielo. br/pdf/er/n36/a08n36.pdf > . Acesso em: 18 jun. 2015.

TABACHNICK, B. G.; FIDELL, L. S. Using multivariate statistics. Boston: Pearson/ Allyn \& Bacon, 2007. 
VISEU, J. et al. Motivação docente: estudo bibliométrico da relação com variáveis individuais, organizacionais e atitudes laborais. Revista Latinoamericana de Psicologia, v. 47, n. 1, 2015. Disponível em: <http:/www.sciencedirect.com/science/article/pii/ S0120053415300078>. Acesso em: 12 dez. 2015.

VLANCHOPOULOS, S. P.; MICHAILIDOUS, S. Development and initial validation of a measure of autonomy, competence, and relatedness in exercise: the basic psychological needs in exercise scale. Measurement in Physical Education and Exercise Science, v. 10, n.3, p. 179-201, 2006.

Texto recebido em 28 de junho de 2016. Texto aprovado em 03 de janeiro de 2017. 